\title{
Rentenversicherung zwischen Nachhaltigkeit und Gerechtigkeit
}

\author{
Friedrich Breyer
}

Zusammenfassung Deutschland hat im Vergleich mit anderen westeuropäischen Staaten bereits weitreichende Schritte unternommen, um die Finanzierung seiner gesetzlichen Rentenversicherung nachhaltig zu sichern. Allerdings sind in den letzten Jahren Bestrebungen zu beobachten, die bereits beschlossenen Rentenreformen wieder rückgängig zu machen. Berücksichtigt man den Zusammenhang zwischen Einkommensniveau und Lebenserwartung, so kann man in der gegenwärtig gültigen Rentenformel in der Tat eine Benachteiligung von Geringverdienern erkennen. Gerade diese Gruppe dürfte unter der 2007 beschlossenen Erhöhung der Regelaltersgrenze überproportional leiden. Um die Nachhaltigkeit der Rentenfinanzierung auch politisch abzusichern, dürfte es daher erforderlich sein, die Rentenformel zugunsten der Geringverdiener abzuändern. Anders ausgedrückt, müssen Nachhaltigkeit und Gerechtigkeit Hand in Hand gehen.

Abstract Compared to other Western European countries, Germany has taken considerable steps to improve the sustainability of financing its public pension system. However, more recently we have observed attempts to revoke some of the enacted reforms before their implementation. If the positive correlation between income and life expectancy is taken into account, one can indeed diagnose a discrimination against low-income earners in the present retirement benefit schedule, and this very group will suffer disproportionately from the increase in legal retirement age enacted in 2007. Therefore, to protect the sustainability of the pension system in the political

\author{
F. Breyer \\ Universität Konstanz und DIW, Berlin, Deutschland \\ F. Breyer $(\bowtie)$ \\ Fachbereich Wirtschaftswissenschaften, Universität Konstanz, Fach 135, 78457 Konstanz, \\ Deutschland \\ e-mail: friedrich.breyer@uni-konstanz.de
}


sphere, it may be necessary to modify the schedule in favor of this group. In other words, sustainability and equity of the pension system must go hand in hand.

\section{Einleitung}

In der gesetzlichen Rentenversicherung hat es in den beiden letzten Jahrzehnten eine Reihe von Reformen gegeben, die die Nachhaltigkeit der Finanzierung angesichts steigender Lebenserwartung und geringer Geburtenzahlen sichern sollten. Insbesondere sind zu nennen:

- die Einführung von Abschlägen bei Renteneintritt vor Erreichen der Regelaltersgrenze mit dem Rentenreformgesetz 1992: dieses Gesetz schwächt die Generosität der 1972 eingeführten ,flexiblen Altersgrenze" ab und regelt, dass für jeden Monat vorzeitiger Inanspruchnahme der Altersrente vor Erreichen der Regelaltersgrenze von derzeit 65 Jahren der Rentenanspruch um 0,3\% sinkt;

- die Berücksichtigung von privaten Altersvorsorge-Beiträgen bei der Berechnung der Entwicklung der verfügbaren Einkommen in der Rentenanpassungsformel („Riester-Treppe") mit dem Rentenreformgesetz 2001: Seitdem richtet sich die Rentenanpassung nach der Steigerung der um Altersvorsorgebeiträge bereinigten Bruttoeinkommen. Die maximal förderungsfähigen Beiträge zur (freiwilligen) Riester-Rente werden dabei wie Pflichtbeiträge behandelt, d.h. in ihrer Einführungsphase von 2003 an sollte der Abzugsbetrag jedes Jahr um 0,5 Prozentpunkte steigen, bis er schließlich im Jahr 20104 Prozent erreicht hätte;

- die Einführung des Nachhaltigkeitsfaktors in der Rentenanpassungsformel, mit der das Zahlenverhältnis von Rentnern zu Beitragszahlern (,Rentnerquotient") berücksichtigt wird, mit dem Rentenversicherungs-Nachhaltigkeitsgesetz von 2004: Durch diesen Faktor ergibt sich ein weiteres Abflachen der (nominalen) Rentenzuwächse um ein Viertel des Prozentsatzes, um den der Rentnerquotient steigt;

- die schrittweise Anhebung der Regelaltersgrenze von 65 auf 67 Jahre zwischen 2012 und 2029 mit dem RV-Altersgrenzenanpassungsgesetz von 2008, und zwar bis 2023 pro Jahr um einen Monat, danach pro Jahr um weitere zwei Monate.

In der letzten Zeit wächst jedoch in der Öffentlichkeit der Unmut über diese Reformen, die als Zumutung für Rentner und Arbeitnehmer angesehen werden, und der Bundestag hat im Vorfeld der Bundestagswahl 2009 bereits zwei Gesetze beschlossen, die die zuvor beschlossenen Maßnahmen teilweise aussetzen, nämlich

- die Verschiebung der ursprünglich auf die Jahre 2007 und 2008 entfallenden Erhöhung des Altersvorsorgeanteils um jeweils 0,5 Prozentpunkte auf die Jahre 2011 und 2012, d.h. die Aussetzung zweier Stufen der „Riester-Treppe": Diese bewirkt eine höhere Rentensteigerung in den jeweiligen Folgejahren 2008 und 2009 und eine Dämpfung der Anpassung in den Jahren 2012 und 2013;

- die „Rentengarantie“ im Gesetz zur Änderung des Vierten Buches Sozialgesetzbuch, zur Errichtung einer Versorgungsausgleichskasse und anderer Gesetze vom 15.7.2009, in der eine Kürzung der nominalen Rentenhöhe im Falle eines Rückgangs des nominellen Lohnniveaus, die nach der bis dahin geltenden Rentenformel im Jahr 2010 eingetreten wäre, für alle Zeiten ausschließt. 
Zudem wird in der SPD offen darüber diskutiert, ob sich die Partei in den kommenden Wahlkämpfen für eine Rücknahme der „Rente mit 67“ einsetzen soll. Dabei wird vor allem auf diejenigen Arbeitnehmer verwiesen, die aus gesundheitlichen Gründen nicht in der Lage seien, bis zu ihrem 67. Lebensjahr zu arbeiten, und für die daher die Erhöhung der Regelaltersgrenze faktisch wie eine Rentenkürzung wirke.

Vor dem Hintergrund dieser politischen Entwicklungen soll der vorliegende Beitrag untersuchen, unter welchen Voraussetzungen es in Deutschland gelingen kann, die bereits beschlossenen Schritte zu mehr Nachhaltigkeit in der Rentenfinanzierung gegen die sich formierende Opposition tatsächlich durchzusetzen. Der Beitrag ist in fünf Thesen gegliedert, die im Folgenden präzisiert und begründet werden sollen.

\section{These 1: Deutschland hat im internationalen Vergleich bereits weitreichende Schritte unternommen, um die Finanzierung seiner gesetzlichen Rentenversicherung nachhaltig zu sichern}

Um die oben genannten Reformschritte, die Deutschland seit der Jahrtausendwende unternommen hat, international einordnen zu können, seien zunächst einzelne Elemente der umlagefinanzierten Rentensysteme verglichen, bevor der Versuch unternommen wird, die Nachhaltigkeitslücken ${ }^{1}$ insgesamt zu betrachten. Als Einzelindikatoren werden dabei verwendet werden:

(1) das gesetzliche Rentenalter,

(2) die Höhe der Abschläge bei vorzeitigem Renteneintritt,

(3) das Rentenniveau bei Erreichen des gesetzlichen Rentenalters,

(4) die Regeln für die Anpassung des allgemeinen Rentenniveaus.

Dabei wird sich der Vergleich auf Westeuropa beziehen, also eine Gruppe von Ländern, die Deutschland in vielerlei Hinsicht ähneln, nicht zuletzt in der Höhe des ProKopf-Produkts.

Um das Ausmaß der Reformen erkennen zu können, seien zunächst die Ausgaben der staatlichen umlagefinanzierten Rentensysteme im Status Quo, also vor Wirksamkeiten der jüngsten Reformen betrachtet. Tab. 1 zeigt, dass Deutschland hinter Italien, Österreich und Frankreich an 4. Stelle unter den 14 betrachteten Nationen liegt. Der Wert von 11,4\% Anteil am BIP liegt nicht nur weit über dem OECD-Durchschnitt von $7,2 \%$, sondern innerhalb der Vergleichsgruppe immerhin doppelt so hoch oder höher als im Vereinigten Königreich, den Niederlanden, Dänemark und Norwegen.

Ad (1): Das gesetzliche Rentenalter, von dem an man ohne Verlust an Rentenansprüchen eine Altersrente beziehen kann, betrug im Jahr 2005 in den meisten betrachteten Ländern 65 Jahre. In Österreich, Italien und dem Vereinigten Königreich galt für Frauen noch das Alter von 60 Jahren. In Frankreich kann man ebenfalls mit 60 Jahren die volle Rente beziehen, allerdings nur nach Ablauf von mindestens 40 Beitragsjahren. Lediglich in Norwegen beträgt die Regelaltersgrenze schon heute 67 Jahre, und neben Deutschland hat nur Dänemark eine schrittweise Anhebung auf 67 Jahre (bis

\footnotetext{
${ }^{1}$ Zum Begriff der Nachhaltigkeitslücke s.u., Ende von These 1.
} 
Tab. 1 Ausgaben für Renten als Anteil am BIP

Quelle: OECD (2009)

\begin{tabular}{lrrrr}
\hline Public cash benefits & \multicolumn{3}{l}{ Per cent of GDP } \\
\cline { 2 - 5 } & 1990 & 1995 & 2000 & 2005 \\
\hline Austria & 11,7 & 12,6 & 12,3 & 12,6 \\
Belgium & 9,1 & 9,3 & 8,9 & 9,0 \\
Denmark & 5,1 & 6,2 & 5,3 & 5,4 \\
Finland & 7,3 & 8,8 & 7,6 & 8,4 \\
France & 10,6 & 12,0 & 11,8 & 12,4 \\
Germany & 10,0 & 10,5 & 11,0 & 11,4 \\
Ireland & 3,9 & 3,5 & 3,1 & 3,4 \\
Italy & 10,1 & 11,4 & 13,6 & 14,0 \\
Netherlands & 6,7 & 5,8 & 5,0 & 5,0 \\
Norway & 5,6 & 5,5 & 4,8 & 4,8 \\
Spain & 7,9 & 9,0 & 8,6 & 8,1 \\
Sweden & 7,7 & 8,2 & 7,3 & 7,7 \\
Switzerland & 5,6 & 6,6 & 6,6 & 6,8 \\
United Kingdom & 4,9 & 5,4 & 5,4 & 5,7 \\
OECD & 6,2 & 6,8 & 6,9 & 7,2 \\
\hline
\end{tabular}

2029 bzw. 2027) bereits beschlossen, während das Vereinigte Königreich eine Anhebung auf 68 Jahre bis zum Jahr 2044 debattiert, allerdings verbunden mit einer Erhöhung des Rentenniveaus. In dieser Frage nimmt Deutschland also eine Vorreiterrolle ein.

Ad (2): Anders sieht es allerdings bei der Höhe der Abschläge bei vorzeitigem Renteneintritt aus (Abb. 1). Hier liegt Deutschland mit 3,6\% pro Jahr des Vorziehens im unteren Teil der Skala. Lediglich die Schweiz und Italien bestrafen vorzeitigen Renteneintritt noch weniger als Deutschland.

Ad (3): Das Niveau der Rentenbezüge ist naturgemäß am schwierigsten zu vergleichen, da es zum einen unterschiedliche Definitionen des Begriffs ,Rentenniveau“ gibt und zum anderen unterschiedliche Abgrenzungen, welche Rentenarten in die Kalkulation einbezogen werden. Der Übersichtlichkeit halber möchte ich mich hier auf die OECD-Daten stützen, nach denen das Rentenniveau als Quotient von Rentenhöhe und Netto-Arbeitseinkommen gemessen wird und obligatorische staatliche Zusatzrenten nach dem Umlagesystem enthalten sind, nicht jedoch kapitalgedeckte Zusatzrenten. Danach war Deutschland das OECD-Land (mit Ausnahme von Portugal), das im Zeitraum von 1990 bis 2005 die stärksten Absenkungen seines Rentenniveaus in der Zukunft beschloss, und zwar um 27 Prozent des Ausgangswerts, wenn man einen Durchschnittsverdiener betrachtet (Martin und Whitehouse 2008, S.12). Demgegenüber wurde etwa im Vereinigten Königreich überhaupt keine Niveauabsenkung und für Geringverdiener sogar eine Steigerung beschlossen.

Im Ergebnis wird Deutschland nach Wirksamwerden aller bereits beschlossenen Reformen im Bruttorentenniveau mit $43 \%$, bezogen auf einen Durchschnittsverdiener, beinahe am unteren Ende der Skala der hier gewählten Vergleichsgruppe liegen (vgl. Tab. 2). Lediglich Irland und das Vereinigte Königreich liegen mit ihrer steu- 


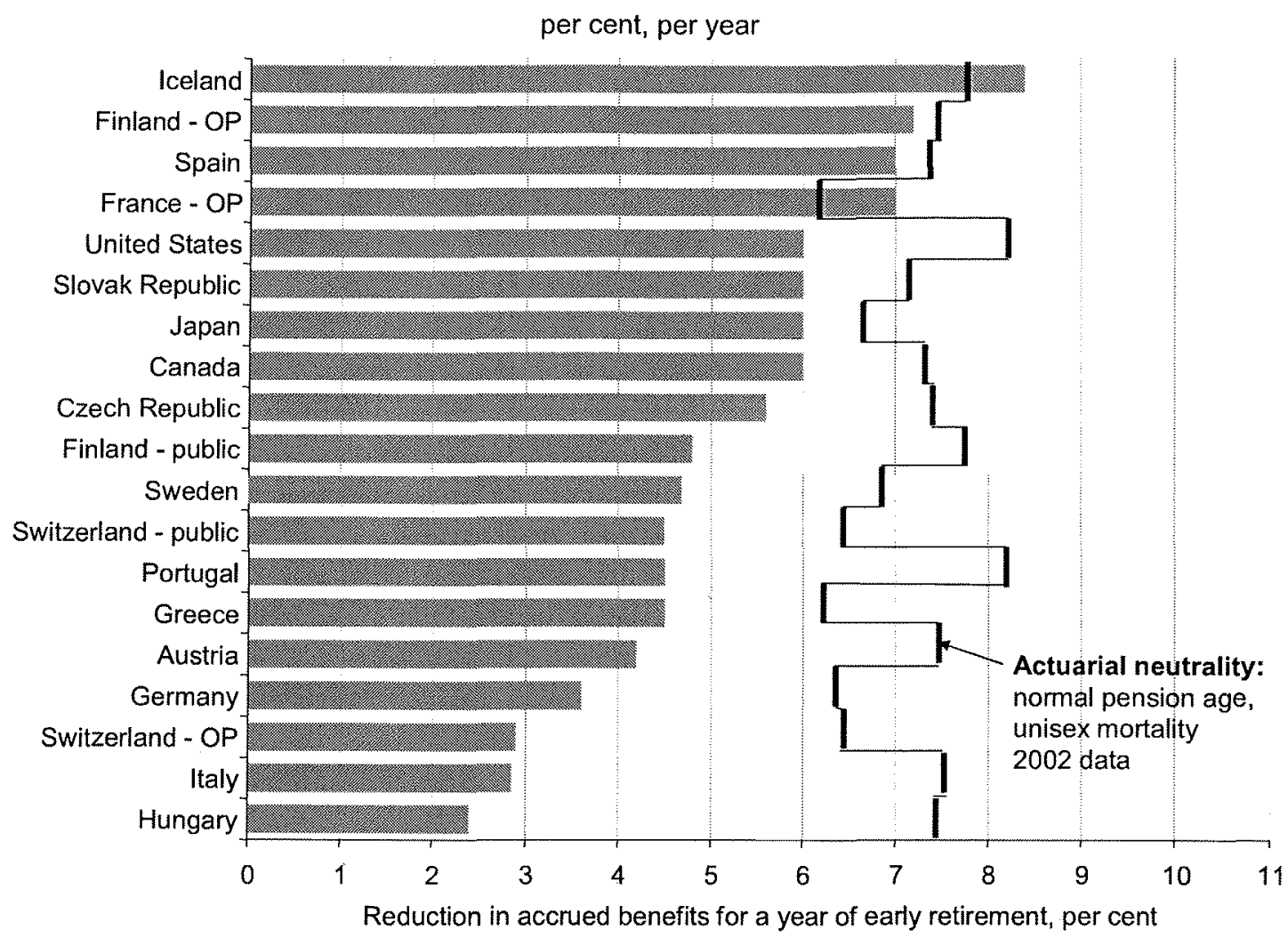

Abb. 1 Abschläge pro Jahr bei vorzeitigem Renteneintritt in der OECD. Quelle: Queisser und Whitehouse (2006), S. 29

Tab. 2 Bruttorentenniveaus

Quelle: OECD (2009)
Gross pension replacement rates by earnings

\begin{tabular}{lrcr}
\hline Men & \multicolumn{3}{c}{ Individual earnings, multiple of mean } \\
\cline { 2 - 4 } & 0,5 & 1 & 1,5 \\
\hline Austria & 80,1 & 80,1 & 76,4 \\
Belgium & 58,1 & 42,0 & 32,5 \\
Denmark & 124,0 & 80,3 & 67,5 \\
Finland & 66,5 & 56,2 & 56,2 \\
France & 61,7 & 53,3 & 48,5 \\
Germany & 43,0 & 43,0 & 42,6 \\
Ireland & 68,4 & 34,2 & 22,8 \\
Italy & 67,9 & 67,9 & 67,9 \\
Netherlands & 93,4 & 88,3 & 86,6 \\
Norway & 66,2 & 59,3 & 49,8 \\
Spain & 81,2 & 81,2 & 81,2 \\
Sweden & 76,6 & 61,5 & 75,6 \\
Switzerland & 62,5 & 58,3 & 40,5 \\
United Kingdom & 51 & 30,8 & 21,3 \\
\hline
\end{tabular}


Tab. 3 Indexierung von Renten in der EU

\begin{tabular}{ll}
\hline Belgien & Preisindex und Entwicklung des allgemeinen Lebensstandards \\
Dänemark & Lohnentwicklung \\
Deutschland & Modifizierte Lohnentwicklung (Nachhaltigkeitsfaktor) \\
Frankreich & Entwicklung der Verbraucherpreise (ohne Tabakerzeugnisse) \\
Griechenland & Entwicklung der Verbraucherpreise \\
Großbritannien & allgemeine Preisentwicklung \\
Italien & Anpassung an Lebenshaltungskosten, allerdings Abstufung: niedrige \\
Luxemburg & Renten zu 100\%, höhere zu 90\% bzw. 75\% \\
Niederlande & Anpassung bei Preissteigerungen von mindestens 2,5\%; ggf. \\
Österreich & Angleichung an Einkommensentwicklung \\
Portugal & Entwicklung der durchschnittlichen Tariflöhne \\
Schweden & Orientierung am Verbraucherpreisindex, teilw. \\
& Pensionistenpreisindex (PIPH) \\
Spanien & Preisentwicklung \\
UK & Garantierte Rente: Preisentwicklung \\
Schweiz & Zusatzrente: durchschnittliche Einkommensentwicklung (max. 1,6\%) \\
& Erwartete Preisentwicklung im Jahr \\
& Entwicklung des allgemeinen Preisniveaus \\
\end{tabular}

Quelle: MISSOC, Sozial Kompass des BMAS

erfinanzierten Grundrente, die für den Durchschnittsverdiener nur 34,2 bzw. 30,8 \% seines Bruttoeinkommens beträgt, darunter. Dagegen zahlen Österreich, Spanien und Dänemark mit ca. $80 \%$ fast doppelt so hohe Renten und auch Italien liegt mit 67,9\% mehr als die Hälfte über dem deutschen Wert. Die Zahl von 88,3\% für die Niederlande ist allerdings mit Vorsicht $\mathrm{zu}$ interpretieren, weil sie nicht nur die staatliche Grundrente, sondern auch die (obligatorische) Betriebsrente enthält.

Diese für die Zukunft prognostizierten Niveauunterschiede sind auch das Resultat unterschiedlicher Anpassungsregeln für die Rentenhöhe. Einige Länder indexieren diese Renten auf die Lohn-, andere auf die Preisentwicklung (vgl. Tab. 3). Deutschland nimmt hier eine Sonderrolle ein, weil in der seit 2005 gültigen Rentenformel neben der Lohnindexierung zwei Komponenten enthalten sind, die die Rentensteigerung bewusst verlangsamen:

1. die „Riester-Treppe“, die über einen geplanten 8-Jahres-Zeitraum die Beiträge zur freiwilligen kapitalgedeckten Zusatzrente rentenmindernd berücksichtigt, und

2. der Nachhaltigkeitsfaktor, der den steigenden Rentnerquotienten ebenfalls rentenmindernd einbezieht.

Da sich ähnliche Regelungen in den Rentenformeln anderer Länder nicht finden, sinkt das deutsche Rentenniveau in den kommenden Jahrzehnten stärker ab als das in den vergleichbaren Ländern.

Überraschender Weise schlagen sich diese Reformen im internationalen Vergleich in der Nachhaltigkeitslücke nicht so stark nieder, wie man vermuten würde 
(vgl. Abb. 2). Die Nachhaltigkeitslücke ist definiert als die Summe aus der expliziten Staatsschuld und dem Barwert der künftigen Netto-Zahlungsverpflichtungen des Staates bei gegenwärtigen Beitrags- und Leistungsniveaus, als Anteil am Bruttoinlandsprodukt pro Jahr (Hagist u.a. 2006, S.5). Sie gibt an, welchen Betrag (bezogen auf das BIP) der Staat zusätzlich in Form von Steuern erheben müsste, um im Zeitablauf einen ausgeglichenen Haushalt zu haben. In Deutschland sinkt die Nachhaltigkeitslücke zwar um gut $100 \%$ des Bruttoinlandsprodukts. Jedoch haben Frankreich und Österreich ihre Nachhaltigkeitslücken durch Rentenreformen um das 3 bis 5-fache reduziert. Raffelhüschen u.a. (2010, S.13) führen dies vor allem auf den Übergang von Lohn- zu Preisindexierung und die Änderung der Berechnungsformel für die Rentenhöhe in diesen beiden Ländern zurück.

\section{These 2: Rentenreformen, die auf eine Kürzung von Rentenansprüchen hinauslaufen, sind politisch schwer durchzusetzen}

Zur Politischen Ökonomie von Rentenreformen gibt es mittlerweile eine sehr umfangreiche Literatur. Einen neueren Überblick geben Galasso und Profeta (2002). Eine der am meisten diskutierten Fragen lautet, wie sich ein Rückgang der Fertilität und ein damit einhergehender Anstieg des Alterslastquotienten, also des Zahlenverhältnisses zwischen Rentnern und Erwerbspersonen, auf die Größe eines Rentensystems, gemessen am Beitragssatz oder am Rentenniveau, auswirkt. Im einfachen Modell einer direkten Demokratie mit einmaliger Abstimmung über ein dauerhaft gültiges Rentensystem (Browning 1975) ist diese Frage nicht eindeutig zu beantworten, denn ein Anstieg des Alterslastquotienten hat zwei gegenläufige Wirkungen: Zum einen verringert er die interne Rendite des Umlageverfahrens und macht daher einen hohen Beitragssatz aus der Sicht eines jungen Wählers weniger attraktiv. Zum anderen erhöht er jedoch das Alter des Medianwählers, und da ein Wähler mit zunehmendem Lebensalter einen immer höheren Beitragssatz wünscht, würde dieser zweite Effekt für sich genommen den Beitragssatz steigern.

In der nachfolgenden Literatur hat es eine Kontroverse zwischen Übelmesser und Sinn (2002) auf der einen und Breyer und Stolte (2001) auf der anderen Seite gegeben. Die erstgenannten Autoren argumentierten im Sinne des Browning-Modells, dass sich in Deutschland das Fenster für nachhaltige Rentenreformen schließen werde, wenn einmal die Mehrzahl der Wähler im Rentenalter oder kurz davor stünden. Die zuletzt genannten Autoren verwiesen dagegen auf die Möglichkeiten der Erwerbspersonen, sich auf andere Weise als an der Wahlurne gegen eine Überforderung durch Rentenbeiträge zu wehren, nämlich durch Flucht in die Selbstständigkeit, in die Schattenwirtschaft oder ins Ausland. Somit würden die Rentner selbst dann, wenn sie die Mehrheit der Wähler stellten, die „Ausbeutung“ der Arbeitnehmer nur so weit treiben, dass sie das Beitragsaufkommen maximierten. Dies wiederum impliziere, dass die Kosten eines weiteren (permanenten) Rückgangs der Fertilität zwischen Beitragszahlern und Rentnern geteilt würden.

Betrachtet man die beiden zuletzt vorgenommenen rentenpolitischen Maßnahmen in Deutschland, nämlich die Aussetzung der Riester-Treppe und die Rentengarantie, so scheinen diese der These von Sinn und Übelmesser nicht nur Recht zu geben, sondern sie sogar zu übertreffen: Obwohl die Rentner im Jahr 2009 noch bei weitem 


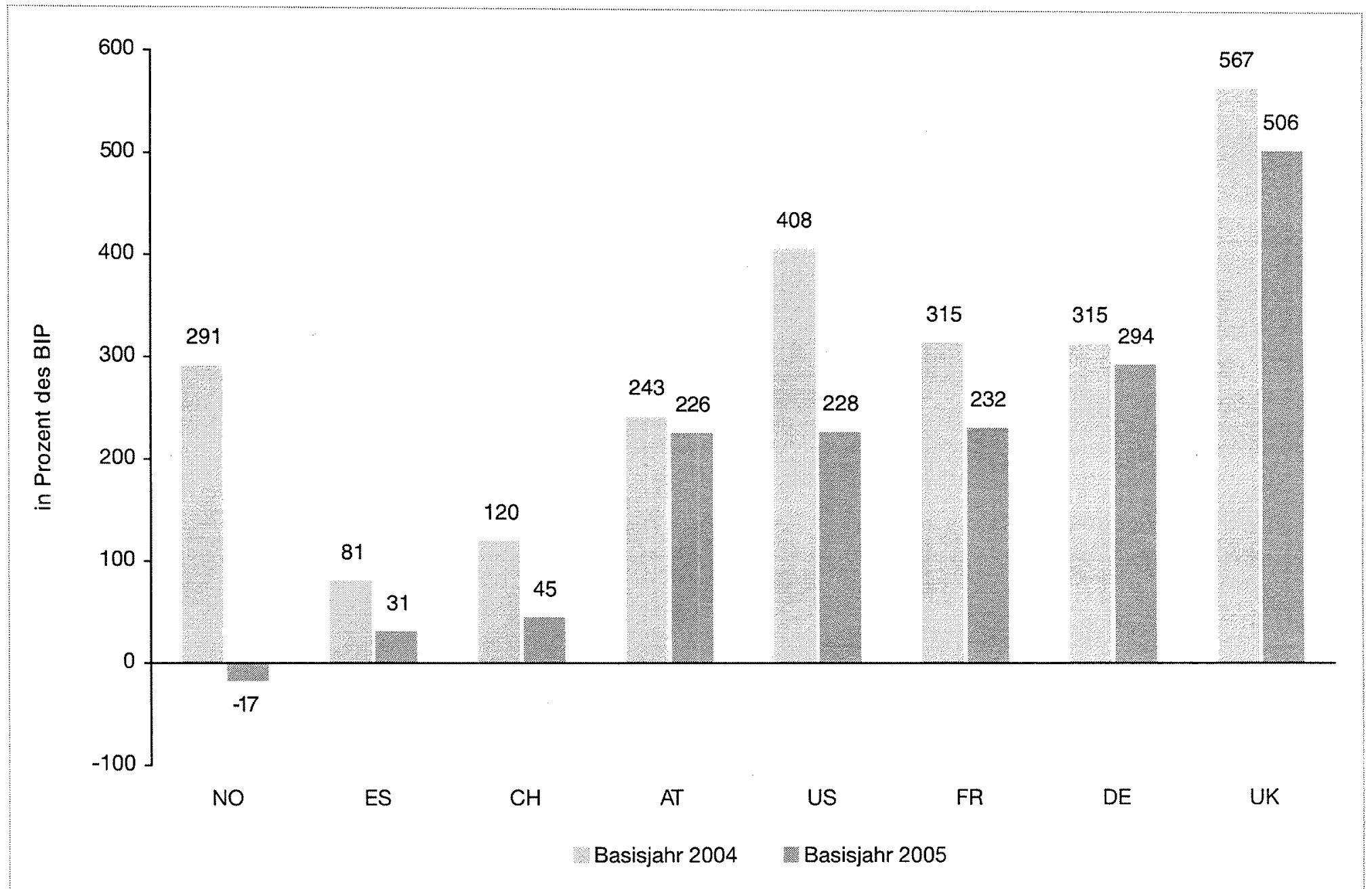

Abb. 2 Änderung der Nachhaltigkeitslücke durch Rentenreformen. Quelle: Raffelhüschen u.a. (2010), S. 12 
nicht die Mehrheit der Wahlberechtigten stellten, fühlten sich die Parteien der damals regierenden Großen Koalition gezwungen, den Rentnern in diesen beiden Punkten entgegen zu kommen, auch wenn dies eine mögliche Beitragssatzsenkung verhinderte. Man kann zudem vermuten, dass diejenige der beiden Volksparteien, die sich gegen die vorgeschlagenen Maßnahmen gestellt hätte, die Bundestagswahl 2009 wohl verloren hätte.

Aus Sicht der Politischen Ökonomie ist ein entsprechendes Wahlverhalten damit zu erklären, dass sich Rentner an der Wahlurne mit ihrer Rolle als Rentner identifizieren. Da die Höhe der Rente für sie - insbesondere für die Bezieher unterdurchschnittlicher Renten - von überragender Bedeutung ist, sind sie in großer Zahl bereit, eine Partei zu wählen, die ihnen in diesem Punkt entgegen kommt, auch wenn sie in anderen politischen Themen eine Präferenz für das Programm einer anderer Partei haben. Demgegenüber ist die Identifikation der Erwerbstätigen mit der Rolle als Beitragszahler - im Gegensatz zu den Annahmen des Browning-Modells - eher schwach ausgeprägt, d.h. die Höhe der Sozialabgaben hat für die Masse der Wähler keine überragende Bedeutung für die Wahlentscheidung.

Die Furcht der politischen Parteien vor einer Abstrafung an der Wahlurne durch die Wähler im Rentenalter, wenn sie zu ihren nachhaltigen Rentenreformen stünden, zeigt jedoch, dass Sinn und Übelmesser in einem anderen Aspekt irrten: Obwohl die Regierenden und das Parlament in der Vergangenheit, wie oben gezeigt, das „Fenster für nachhaltige Rentenreformen" genutzt haben, folgt daraus noch lange nicht, dass diese Reformen unumkehrbar sind, denn die Entwicklungen im Zuge der Bundestagswahl 2009 zeigen, dass es die Macht der Rentner an der Wahlurne durchaus schafft, eine einmal beschlossene und sogar begonnene Rentenreform wieder rückgängig zu machen.

\section{These 3: Betrachtet man den Zusammenhang zwischen Einkommensniveau und Lebenserwartung, so kann man in der gegenwärtig gültigen Rentenformel eine Benachteiligung der Geringverdiener erkennen}

Einer der Grundpfeiler der Gesetzlichen Rentenversicherung in Deutschland ist das Prinzip der Teilhabe-Äquivalenz. Es besagt, dass sich die Rentenansprüche zweier Rentner, die im selben Jahr geboren sind und gleichzeitig aus dem Arbeitsleben ausscheiden, genau proportional zu den von ihnen insgesamt gezahlten Beiträgen verhalten. Bezog einer immer das doppelte Einkommen des anderen (und zahlte er daher die doppelten Beiträge), so ist seine monatliche Rente doppelt so hoch wie die des anderen. Hierdurch soll eine systematische Einkommensumverteilung in der Rentenversicherung vermieden werden. ${ }^{2}$ Anders ausgedrückt, ist jeder Beitrags-Euro in der GRV gleich viel wert.

\footnotetext{
${ }^{2}$ Hierbei muss aber angemerkt werden, dass in der GRV in verschiedener Weise Umverteilung betrieben wird. So gibt es eine rentenrelevante Anrechnung von Kindererziehungszeiten oder auch von Zeiten der Ausbildung. Zudem findet eine Umverteilung im Falle von Erwerbsminderung statt. Diese versicherungsfremden Leistungen werden aber zumindest offiziell nicht aus Beiträgen, sondern durch den steuerfinanzierten Bundeszuschuss zur GRV finanziert.
} 
Der Mangel dieses Konzepts besteht allerdings darin, dass allein die monatlichen Rentenansprüche in einer festen Relation zu den insgesamt gezahlten Beiträgen stehen. Eine Aussage zur Verteilungs-Neutralität kann aber erst dann getroffen werden, wenn die gesamte (erwartete) Rentenleistung zu den insgesamt gezahlten Beiträgen ins Verhältnis gesetzt wird. Die gesamte Rentenleistung hängt allerdings neben der monatlichen Rente auch von der erwarteten Bezugsdauer der Rente ab, die wiederum von der Lebenserwartung der entsprechenden Einkommensgruppe bestimmt wird. In zahlreichen Studien wurde zudem nachgewiesen, dass die Lebenserwartung systematisch mit steigendem Einkommen zunimmt. ${ }^{3}$

In einer neueren Studie haben Breyer und Hupfeld (2009) einen großen Datensatz der GRV über 382.000 männliche Rentner, die zwischen 1994 und 2005 gestorben sind, analysiert ${ }^{4}$ und konnten zeigen, dass ein systematischer (und zwar positiver) Zusammenhang zwischen der Höhe der erworbenen Entgeltpunkte pro Jahr (als Indikator für das Einkommen) und der Lebenserwartung besteht: Spezifiziert man den Zusammenhang zwischen Einkommen (Y) und Lebenserwartung (L) als linear, so lautet der geschätzte Zusammenhang ${ }^{5}$ :

$$
L=70,17+4,05 \cdot Y,
$$

d.h. ein zusätzlicher Entgeltpunkt pro Jahr ist mit einer um ca. vier Jahre längeren Lebenserwartung verbunden.

Daraus folgt aber unmittelbar, dass die Rendite eines Beitrags-Euros, gemessen am erwarteten Rentenanspruch, umso höher ist, je mehr Entgeltpunkte pro Jahr gesammelt wurden. Das Rentensystem verteilt also tatsächlich um, und zwar zu Gunsten der Gruppen mit überdurchschnittlicher Lebenserwartung, und dies sind nach (1) die Besserverdienenden.

\section{These 4: Gerade die Gruppe der Geringverdiener dürfte unter einer Erhöhung der Regelaltersgrenze überproportional leiden}

Dass die Erhöhung der Regelaltersgrenze die verschiedenen Einkommensgruppen sehr unterschiedlich trifft, kann man sich an (1) klarmachen, wenn man z.B. einen Geringverdiener, der pro Jahr 0,5 Entgeltpunkte sammelt, mit einem Besserverdiener vergleicht, der jährlich 1,5 Entgeltpunkte bezieht. Deren Lebenserwartungen betragen 72,2 bzw. 76,2 Jahre. Damit sinkt die erwartete Rentenbezugsdauer im ersten Fall von 7,2 auf 5,2, im zweiten Fall von 11,2 auf 9,2 Jahre. Während der Besserverdiener somit durch die Erhöhung der Regelaltersgrenze 18 Prozent seiner erwarteten Bezugsdauer einbüßt, macht die Reform bei dem betrachteten Geringverdiener fast 28 Prozent aus.

\footnotetext{
${ }^{3}$ Vergleiche etwa Reil-Held (2000) und Gaudecker und Scholz (2007) für Deutschland sowie Cutler u.a. (2006) für die USA.

${ }^{4}$ Vergleiche FDZ-RV (2007).

${ }^{5}$ Die Hinzunahme eines quadratischen Einkommens-Terms trägt nur wenig zur Erklärung der Lebenserwartung bei und ändert den Koeffizienten des linearen Terms kaum.
} 
Von den politischen Gegnern der „Rente mit 67“" werden auch regelmäßig Arbeitnehmer in Berufsgruppen angeführt, die mit schwerer körperlicher Arbeit verbunden sind, so der sprichwörtliche Dachdecker. Ein Grund für die Schwierigkeiten, die diese Personengruppe durch eine Erhöhung der Regelaltersgrenze erfahren dürfte, liegt wohl in der mangelnden beruflichen Flexibilität gerade derer, die keine akademische Ausbildung erhalten haben. Ein Wechsel in eine körperlich weniger belastende Tätigkeit ist gegen Ende des Berufslebens kaum noch möglich. Hier müsste, wenn Frühverrentung vermieden werden soll, eine langfristige Karriereplanung mit frühzeitigem Umstieg in eine körperlich leichtere Tätigkeit erfolgen.

\section{These 5: Um die Nachhaltigkeit der Rentenfinanzierung auch politisch abzusichern, dürfte es erforderlich sein, die Rentenformel zugunsten der Geringverdiener abzuändern. Anders ausgedrückt, müssen Nachhaltigkeit und Gerechtigkeit Hand in Hand gehen}

Aus dem oben Gesagten lässt sich ableiten, dass die Nachhaltigkeit der Rentenfinanzierung in Deutschland politisch gefährdet ist. Die Erfahrung der vergangenen Jahre zeigt, dass sich mit dem Abrücken von zuvor beschlossenen, aber noch nicht implementierten Rentenreformen Wählerstimmen gewinnen lassen. Dabei wird in der politischen Debatte insbesondere auf die Benachteiligung von Angehörigen harter Berufe mit geringem Einkommen verwiesen. Um die Nachhaltigkeit zu sichern, wird es daher unumgänglich sein, eine Abmilderung der Reformwirkungen für diese Bevölkerungsgruppe vorzunehmen. Dazu gibt es im Prinzip drei Strategien:

(a) eine Differenzierung der Regelaltersgrenze nach Berufs- oder Einkommensgruppen,

(b) eine Senkung der Abschläge bei vorzeitigem Rentenbezug, oder

(c) eine Änderung der gesamten Rentenformel zu Gunsten der Geringverdiener.

Ad (a): In der politischen Debatte wird verstärkt die Einführung einer vorgezogenen Altersgrenze für bestimmte Berufsgruppen gefordert. Schon jetzt gilt für Personen, die 45 Jahre als Pflichtbeitrags- oder Kindererziehungszeiten vorzuweisen haben (,besonders langjährig Versicherte“), die Altersgrenze von 65 Jahren für eine abschlagsfreie Rente fort. Da man auf die 45 Jahre nur dann kommen kann, wenn man spätestens mit 20 Jahren ins Erwerbsleben eingetreten ist, sind von dieser Regelung Akademiker im Wesentlichen ausgenommen. Ansonsten gibt es jedoch keine Differenzierung nach Einkommen oder Berufsgruppe. Ein Bankmanager, der seit seiner Banklehre ununterbrochen erwerbstätig war, profitiert gleichfalls von dieser Regelung.

Wollte man allerdings zusätzliche Ausnahme-Tatbestände schaffen, etwa für den sprichwörtlichen Dachdecker oder andere Berufe, so hat das zwei gravierende Nachteile: zum einen drohen die Ausnahmen uferlos zu werden, so dass das Prinzip der „Rente mit 67“ ausgehöhlt werden könnte, und zum anderen öffnet die Fokussierung auf Berufsgruppen dem Lobbyismus Tür und Tor. Am Ende werden nicht diejenigen Gruppen begünstigt, die eine besonders kurze Lebenserwartung haben, sondern die, die sich besonders leicht organisieren lassen und daher politisch Gehör finden können. Von diesem Weg ist also dringend abzuraten. 
Ad (b): Geht man davon aus, dass mit einem niedrigen Einkommen und unterdurchschnittlicher Lebenserwartung auch größere gesundheitliche Probleme in den Jahren vor Erreichen der Altersgrenze verbunden sind, so könnte man vermuten, dass die betreffende Gruppe von Arbeitnehmern davon besonders profitieren würde, wenn die Abschläge von der Rentenhöhe bei vorzeitigem Renteneintritt wieder verringert würden. Breyer und Hupfeld (2010) sind der Frage nachgegangen, ob man auf diese Weise die Ungleichheit in der Rendite des Rentensystems zwischen den Einkommensgruppen dämpfen könnte. Dazu haben sie einen Datensatz der Rentenversicherung untersucht, der eine 10-Prozent-Stichprobe aller männlichen Versicherten umfasst, die im Zeitraum 1992 bis 2004 verstorben sind. Gefragt wurde, bei welchem Wert des prozentualen Rentenabschlags, der derzeit 3,6 \% im Jahr beträgt, die interne Rendite des Rentensystems nicht mehr vom jährlichen Einkommen (bzw. den Entgeltpunkten) des Versicherten abhängt. Das überraschende Resultat ist, dass man dazu in der betreffenden Sterbekohorte die Abschläge noch hätte erhöhen müssen (auf 5,9 Prozent pro Jahr). Eine Erklärung dafür könnte darin bestehen, dass in der betrachteten Kohorte besser verdienende Versicherte sogar eher in Rente gegangen sind. Dies wiederum dürfte die Folge der massiven Subventionierung der Frühverrentung in den 80er und 90er Jahren gewesen sein, von der überwiegend große Unternehmen Gebrauch gemacht haben, die üblicher Weise auch höhere Löhne zahlen. ${ }^{6}$

Daneben hätte eine Senkung der Rentenabschläge auch noch negative Anreizwirkungen, da sie die Frühverrentung wieder attraktiver machen und somit die Fortschritte in der Nachhaltigkeit der Rentenfinanzierung gefährden würde. Auch von diesem Weg muss also abgeraten werden.

Ad (c): Es verbleibt schließlich die Besserstellung der Geringverdiener in der Rentenformel insgesamt. Dies hat zwar keine direkten Auswirkungen auf die Regelaltersgrenze, verschafft aber bei der betroffenen Bevölkerungsgruppe den finanziellen Spielraum, der es dem Einzelnen ermöglicht, vorzeitig in den Ruhestand zu gehen und den Rentenabschlag in Kauf zu nehmen.

Breyer und Hupfeld (2009) haben auf der Grundlage der (1) errechnet, wie die Rentenformel budgetneutral modifiziert werden müsste, damit das Verhältnis zwischen (auf Grund der Lebenserwartung erwarteten) Rentenanspruch und insgesamt gezahlten Beiträgen vom Einkommen unabhängig wäre. Die dort vorgeschlagene Formel lautet:

$$
R_{t}\left(P_{i}\right)=V_{t} \cdot P_{i} \cdot T \cdot \frac{10.16}{5.17+4.05 \cdot P_{i}} .
$$

Darin bezeichnet $R_{t}$ die (monatliche) Rentenhöhe, $P_{i}$ die vom Versicherten $i$ erworbenen Entgeltpunkte, $T$ seine Versicherungsdauer und $V_{t}$ die volkswirtschaftliche Komponente der Rentenhöhe im Jahr $t$. Die gegenwärtig gültige Rentenformel umfasst nur die ersten drei Terme auf der rechten Seite der Gleichung. Neu ist der von den Autoren vorgeschlagene Korrekturfaktor, der Bruch auf der rechten Seite. Darin drückt der Zähler die durchschnittliche Lebenserwartung eines männlichen 65jährigen Rentenversicherten aus und der Nenner den in (1) dargestellten, empirisch

\footnotetext{
${ }^{6}$ Ein anderer Grund dafür, dass die besser Verdienenden eher in Rente gegangen sind, könnte auch der Einkommenseffekt sein, dass diese mehr Geld sparen konnten bzw. eine bessere zusätzlich private Altersvorsorge hatten.
} 
Tab. 4 Auswirkungen der neuen Rentenformel

\begin{tabular}{|c|c|c|c|}
\hline Jahreseinkommen & Jahresrente (alt) & Jahresrente (neu) & Relation neu alt \\
\hline 0 & 0 & 0 & \\
\hline 2.400 & 1.003 & 1.853 & 1,85 \\
\hline 4.800 & 2.006 & 3.497 & 1,74 \\
\hline 7.200 & 3.010 & 4.965 & 1,65 \\
\hline 9.600 & 4.013 & 6.283 & 1,57 \\
\hline 12.000 & 5.016 & 7.475 & 1,49 \\
\hline 14.400 & 6.019 & 8.556 & 1,42 \\
\hline 16.800 & 7.022 & 9.542 & 1,36 \\
\hline 19.200 & 8.026 & 10.444 & 1,30 \\
\hline 21.600 & 9.029 & 11.274 & 1,25 \\
\hline 24.000 & 10.032 & 12.039 & 1,20 \\
\hline 26.400 & 11.035 & 12.747 & 1,16 \\
\hline 28.800 & 12.038 & 13.403 & 1,11 \\
\hline 31.200 & 13.041 & 14.014 & 1,07 \\
\hline 33.600 & 14.045 & 14.583 & 1,04 \\
\hline 36.000 & 15.048 & 15.116 & 1,00 \\
\hline 38.400 & 16.051 & 15.615 & 0,97 \\
\hline 40.800 & 17.054 & 16.083 & 0,94 \\
\hline 43.200 & 18.057 & 16.523 & 0,92 \\
\hline 45.600 & 19.061 & 16.939 & 0,89 \\
\hline 48.000 & 20.064 & 17.330 & 0,86 \\
\hline 54.000 & 22.572 & 18.220 & 0,81 \\
\hline 60.000 & 25.080 & 19.001 & 0,76 \\
\hline 64.800 & 27.086 & 19.559 & 0,72 \\
\hline
\end{tabular}

Quelle: eigene Berechnungen

ermittelten Zusammenhang zwischen der erwarteten Bezugsdauer und der Zahl an Entgeltpunkten pro Jahr. ${ }^{7}$

Tab. 4 enthält die Ergebnisse einer Modellrechnung, der die (für Männer) durchschnittliche Zahl von Beitragsjahren $(39,1)$ und der Wert eines Entgeltpunkts von 26,27 Euro (aus dem Jahr 2007) zugrunde liegen. In der Konsequenz werden alle Renten unterhalb von 15.000 Euro im Jahr (die einem Jahreseinkommen von 36.000 Euro entsprechen), aufgewertet, und zwar prozentual um so stärker, je niedriger das Jahreseinkommen ist, wobei die Aufwertung bei sehr kleinen Renten gegen den Faktor 1,965 konvergiert. Umgekehrt werden hohe Renten verringert, was bei der Maximalrente, die einem Einkommen an der Beitragsbemessungsgrenze über 40 Jahre entspricht, einen Abschlag von $28 \%$ ausmacht.

\footnotetext{
${ }^{7}$ Da die Datenanalyse ausschließlich männliche Versicherte umfasste, kann sich auch die Rentenformel nur auf Männer beziehen.
} 
Breyer und Hupfeld (2009) können ferner zeigen, dass die vorgeschlagene Rentenformel eine erhebliche Wirkung auf die Höhe der Altersarmut unter langjährigen Beitragszahlern hätte. Dazu verwenden sie ebenfalls Daten der GRV, nämlich eine 10Prozent-Stichprobe aller Rentenzahlungen an männliche Altersrentner im Jahr 2004, die mindestens 35 Beitragsjahre aufweisen, insgesamt knapp 40.000 Fälle.

Für diese wurde zunächst der Anteil der Fälle ermittelt, in denen der monatliche Rentenzahlbetrag niedriger war als die Höhe der „Grundsicherung im Alter“ (von damals 589 Euro/Monat). Dies ist zumindest für alleinstehende Rentner ohne sonstiges Einkommen ein relevanter Vergleich. Es ergibt sich, dass sich durch die vorgeschlagene Rentenformel der Anteil der Bezieher von Renten unterhalb des Grundsicherungsniveaus - der im Jahr 2004 mit 1,2 Prozent noch sehr gering war - um mehr als drei Viertel auf knapp 0,26 Prozent verringert hätte. Unterstellt man, dass das Rentenniveau - wie bis 2030 vorgesehen - um 15 Prozent sinkt, so wäre der Anteil der Rentenbezieher unterhalb der Grundsicherung mit 2,4 Prozent absolut höher. Durch die hier vorgeschlagene Reform würde deren Anteil aber ebenso stark auf 0,56 Prozent abnehmen.

Die Autoren schließen daraus, dass auf ein weiteres, teures Instrument zur Bekämpfung der Altersarmut - neben der Grundsicherung im Alter - verzichtet werden kann, wenn mit dem Prinzip der Verteilungsneutralität im deutschen Rentensystem ernst gemacht wird: Bezieht man die Lebenserwartung der verschiedenen Einkommensgruppen in die Berechnung der Rentenansprüche ein und beendet damit die Umverteilung zu Gunsten der Besserverdienenden auf Grund ihrer längeren Rentenbezugszeiten, so lässt sich auch der drohenden Altersarmut unter langjährigen Beitragszahlern wirksam begegnen.

\section{Fazit}

Nach den Rentenreformen des vergangenen Jahrzehnts konnte man den Eindruck gewinnen, dass Deutschland auf diesem Feld der Sozialpolitik bereits genug unternommen hat, um angesichts der absehbaren demographischen Entwicklung eine langfristig nachhaltige Finanzierung zu sichern. In der Tat sind vor allem die Absenkung des Rentenniveaus und die Verlängerung der (gesetzlichen) Lebensarbeitszeit im internationalen Vergleich beachtlich. Allerdings sind alle diese Rentenreformen in Gefahr, ausgesetzt, zeitlich gestreckt oder sogar rückgängig gemacht zu werden, wenn die Parteien vor wichtigen Wahlen um die Gunst der Wählerstimmen der wachsenden Zahl von Rentnern werben. Besonders unpopulär ist die Hinausschiebung der Regelaltersgrenze auf 67 Jahre, und noch hat der Zeitraum der Implementierung (2012 bis 2029) nicht begonnen. Besonders auf der politischen Linken mehren sich die Stimmen, diese „unsoziale“ Reform nicht zu implementieren, da sie vor allem den Angehörigen körperlich anstrengender Berufe eine unzumutbare Härte auferlege. Berücksichtigt man den gut gesicherten empirischen Zusammenhang zwischen Einkommen und Lebenserwartung, so ist dieser Einwand nicht ganz von der Hand zu weisen. Um die Implementierung der beschlossenen Rentenreformen politisch abzusichern, erscheint es erforderlich, die Rentenformel zugunsten der Geringverdiener abzuändern. Ohne mehr „Gerechtigkeit“ im Rentensystem könnte auch die Nachhaltigkeit in Gefahr sein. 
Danksagung Für wertvolle Hinweise danke ich Benjamin Groth, Verena Lauber und Dominik Sachs.

\section{Literatur}

Breyer, F., Hupfeld, S.: Fairness of public pensions and old-age poverty. Finanz Archiv 65, 358-380 (2009)

Breyer, F., Hupfeld, S.: On the fairness of early retirement provisions. Ger. Econ. Rev. 11, 60-77 (2010)

Breyer, F., Stolte, K.: Demographic change, endogenous labor supply, and the feasibility of pension reform. J. Popul. Econ. 14, 409-424 (2001)

Browning, E.K.: Why the social insurance budget is too large in a democracy. Econ. Inq. 13, 373-388 (1975)

Cutler, D., Deaton, A., Lleras-Muney, A.: The determinants of mortality. J. Econ. Perspect. 20, 97-120 (2006)

FDZ-RV: Scientific Use File Demografiedatensatz Rentenwegfall 1993-2005. SUFRTWFijXVSTDemo, Data Set from Deutsche Rentenversicherung Bund (2007)

Galasso, V., Profeta, P.: The political economy of social security. Eur. J. Polit. Econ. 18, 1-29 (2002)

Gaudecker, H.-M., Scholz, R.D.: Differential mortality by lifetime earnings in Germany. Demogr. Res. 17, 83-108 (2007)

Hagist, C., Raffelhüschen, B., Weddige, O.: Brandmelder der Zukunft - Die Generationenbilanz 2004. Forschungszentrum Generationenverträge, Albert-Ludwigs-Universität Freiburg, Freiburg (2006)

Martin, J.P., Whitehouse, E.: Reforming retirement-income systems: lessons from the recent experiences of OECD countries. OECD Social Employment and Migration Working Papers, OECD, Paris (2008)

OECD: Pensions at a Glance. OECD, Paris (2009)

Queisser, M., Whitehouse, E.: Neutral or fair? Actuarial concepts and pension-system design. OECD Social, Employment and Migration Working Papers No. 40, OECD, Paris (2006)

Raffelhüschen, B., Moog, S., Müller, C.: Ehrbare Staaten? Die deutsche Generationenbilanzierung im internationalen Vergleich: Wie gut ist Deutschland auf die demographische Herausforderung vorbereitet? Stiftung Marktwirtschaft, Argumente zu Marktwirtschaft und Politik Nr. 110, Mai (2010)

Reil-Held, A.: Einkommen und Sterblichkeit in Deutschland: Leben Reiche länger? Beitr. Angew. Wirtsch.forsch., No. 580-00 (2000)

Übelmesser, S., Sinn, H.-W.: Pensions and the path to gerontocracy in Germany. Eur. J. Polit. Econ. 19, 153-158 (2002) 